\title{
El aborto y la educación médica en México
}

\author{
Deyanira González de León-Aguirre, MSP,(I) \\ Deborah L Billings, Dra en Soc, ${ }^{(2)}$ Rubén Ramírez-Sánchez, MC. ${ }^{(3)}$
}

González de León-Aguirre D, Billings DL, Ramírez-Sánchez R. El aborto y la educación médica en México. Salud Publica Mex 2008;50:258-267.

\begin{abstract}
Resumen
La educación médica en México tiene deficiencias importantes en el terreno de la salud sexual y reproductiva, $y$ ofrece a los estudiantes pocos elementos para el reconocimiento del aborto como un problema relevante de la práctica profesional de la medicina. La educación médica carece de opciones para la capacitación clínica de los futuros médicos en modelos para la atención integral del aborto, que incluyen el uso de tecnologías seguras y eficaces, y una gama de servicios para responder a las necesidades de las mujeres. Estas limitaciones tienen especial relevancia en países como México, donde el aborto inseguro sigue siendo un importante problema de salud pública.Además, el marco legal del aborto ha empezado a cambiar en la década presente, por lo que la búsqueda de alternativas para incorporar un enfoque amplio sobre el aborto en los programas de las escuelas de medicina es una tarea que no se puede postergar.
\end{abstract}

Palabras clave: educación médica; aborto; salud sexual y reproductiva; México
González de León-Aguirre D, Billings DL, Ramírez-Sánchez R. Abortion and medical education in Mexico. Salud Publica Mex 2008;50:258-267.

\section{Abstract}

Medical education in Mexico has significant deficiencies in the area of sexual and reproductive health and does not offer students the information needed for dealing with abortion as a relevant problem in the professional practice of medicine. Medical education does not offer options for the clinical training of future physicians in integrated models for abortion care, which include the use of safe and effective technologies as well as a range of services to respond to women's needs. These limitations are especially relevant in countries such as Mexico where unsafe abortion continues to be a significant public health problem. In addition, the legal context for abortion has begun to change during the current decade; therefore, the search for alternatives to incorporate a broad approach to abortion in medical school programs is a task that cannot be postponed.

Key words: medical education; abortion; sexual and reproductive health; Mexico
— ste trabajo se refiere a la necesidad de incorporar una ـperspectiva amplia sobre el aborto en la educación médica, un tema que ha merecido escasa atención en México. La formación de los médicos ofrece pocos elementos para entender la diversidad de motivos por los cuales las mujeres interrumpen sus embarazos; para ubicar al aborto como un problema relevante de la práctica de la medicina; para reconocer sus vínculos con la salud pública, la desigualdad social y los derechos de las mujeres; y para comprender sus implicaciones éticas y legales. La formación de los médicos carece también de opciones para su capacitación en modelos

(I) Profesora-investigadora, Universidad Autónoma Metropolitana-Xochimilco.

(2) Investigadora, Ipas Global.

(3) Director Médico de Ipas México.

Fecha de recibido: 17 de agosto de 2007 - Fecha de aceptado: 30 de enero de 2008

Solicitud de sobretiros: Deyanira González de León-Aguirre. Departamento de Atención a la Salud, Universidad Autónoma Metropolitana-Xochimilco. Calzada del Hueso I 100, Col.Villa Quietud. 04960 Delegación Coyoacán, México DF.

Correo electrónico: dgonzal@correo.xoc.uam.mx, deyagla@yahoo.com.mx 
para la atención integral del aborto, que incluyen el uso de tecnologías seguras y ofrecen a las mujeres servicios que abarcan una amplia gama de necesidades.

El artículo se apoya en la experiencia de sus autores en la formación de personal de salud en el tema del aborto y en la implementación de un proyecto de colaboración entre Ipas México y una selección de escuelas de medicina y enfermería de universidades públicas. ${ }^{*} \mathrm{El}$ proyecto inició en 2001 para apoyar a las universidades en el reforzamiento de sus programas en el campo de la salud sexual y reproductiva (SSR) y en la incorporación de un enfoque que incluya las distintas dimensiones del aborto. ${ }^{1}$

La salud reproductiva de las mujeres constituyó el tema central de discusión en la Conferencia Internacional de Población y Desarrollo (CIPD), convocada en 1994 por las Naciones Unidas en El Cairo. La importancia de la CIPD radica en que sus recomendaciones -aprobadas por 179 gobiernos, organizaciones de mujeres, agencias financieras y organismos internacionales- proporcionaron el marco de referencia necesario para reorientar las políticas de población en los países en desarrollo y abordar los problemas del campo de la SSR desde un enfoque conceptual novedoso y progresista. ${ }^{2,3}$ Por otro lado, la CIPD fue el primer foro mundial que logró un consenso sin precedentes respecto a la importancia social y sanitaria del aborto inseguro; el Programa de Acción de esta conferencia convocó a los gobiernos a asumir su responsabilidad frente al problema y precisó las medidas a ser adoptadas: ${ }^{4}$

Se insta a todos los gobiernos y a las organizaciones intergubernamentales y no gubernamentales pertinentes a incrementar su compromiso con la salud de la mujer, a ocuparse de los efectos que en la salud tienen los abortos realizados en condiciones no adecuadas como un importante problema de salud pública [...] Se debe asignar siempre máxima prioridad a la prevención de los embarazos no deseados y habrá que hacer todo lo posible para eliminar la necesidad del aborto [...] En los casos en

\footnotetext{
* Ipas es una organización no gubernamental que trabaja en el ámbito internacional. Las actividades de Ipas en México iniciaron hace más de 20 años e incluyen la promoción de la salud y los derechos reproductivos de las mujeres, y la capacitación de personal en modelos integrales para la atención del aborto (ver: www.ipas.org). Ipas ha colaborado con las siguientes universidades: Universidad Nacional Autónoma de México, Universidad Autónoma Metropolitana, Universidad Veracruzana, Universidad Autónoma de Oaxaca, y Universidad de Tlaxcala. Una actividad central del proyecto ha sido la organización de talleres extracurriculares sobre el aborto, cuya evaluación ha sido positiva por parte de los estudiantes y los académicos responsables en cada escuela.
}

que el aborto no es contrario a la ley, los abortos deben realizarse en condiciones adecuadas. En todos los casos, las mujeres deben tener acceso a servicios de calidad para tratar las complicaciones derivadas del aborto. Se deben ofrecer con prontitud servicios de planificación de la familia, educación y asesoramiento post aborto que ayuden también a evitar la repetición de los abortos (párrafo 8.25).

En 1999, durante la primera evaluación del Programa de Acción de la CIPD, los gobiernos participantes refrendaron su compromiso de mejorar la atención a las mujeres con complicaciones del aborto y de garantizar el acceso a servicios seguros para la interrupción del embarazo en las circunstancias amparadas por las leyes: ${ }^{5}$

... en circunstancias en las que el aborto no está en contra de la ley, los sistemas de salud deben capacitar y equipar a los proveedores de servicios de salud y tomar medidas que aseguren que el aborto es seguro y accesible. Medidas adicionales deben ser tomadas para salvaguardar la salud de las mujeres (párrafo 63.iii).

A partir de los compromisos asumidos por el gobierno mexicano en estas conferencias, la Secretaría de Salud (SSA) creó en 1994 el Programa Nacional de Salud Reproductiva, sustentado en los lineamientos de El Cairo y de la Reforma del Sector Salud del país, así como en las políticas nacionales de población y las dirigidas a mejorar la salud y la condición social de las mujeres. ${ }^{6}$ En 2001, se publicó el Programa de Acción: Salud Reproductiva, cuyos principios rectores son el derecho a la información, el respeto a los derechos sexuales y reproductivos y el derecho a los servicios de salud. ${ }^{7}$ Sin embargo, el enfoque de la SSR ha sido insuficientemente incorporado a los programas de las licenciaturas en medicina, lo cual refleja una escasa vinculación entre la educación médica y las políticas públicas. Además, el intenso debate sobre el aborto que se ha generado en las últimas décadas en el ámbito mundial y nacional no ha impactado la formación de los médicos, lo que adquiere especial importancia frente a las reformas que se han aprobado en México para liberalizar las leyes que lo regulan.

El aborto es un problema relevante de salud pública en México, cuyos efectos adversos son propiciados por las condiciones de inseguridad en que generalmente se practica. Los médicos tienen un papel central para asegurar el acceso de las mujeres al aborto en las circunstancias en que es legal, así como la responsabilidad de garantizar una atención de alta calidad a quienes sufren complicaciones del aborto inseguro. Por ello, incorporar 
el enfoque de la SSR a la educación médica, con una perspectiva amplia que incluya la atención integral del aborto, es una tarea que no se puede evadir ni se debe postergar.

\section{Leyes sobre el aborto en México}

El debate sobre el aborto en México ha sido obstaculizado por la iglesia católica y otros grupos conservadores -como el Comité Nacional Pro-Vida y el Partido Acción Nacional- que han propiciado la confusión y el amarillismo sobre el tema. En los últimos años, sin embargo, gracias a importantes cambios en la vida política del país, la discusión sobre el aborto ha adquirido nuevos matices y la antigua demanda feminista para liberalizar las leyes ha ganado el apoyo de los sectores progresistas y una mayor atención entre la sociedad civil. ${ }^{8,9}$ Como resultado de los esfuerzos para dar visibilidad a la situación del aborto, el marco legal que lo regula ha empezado a cambiar de manera sustantiva en años recientes.

Salvo en la capital del país, las leyes sobre el aborto en México son restrictivas y la mayoría de las legislaciones estatales carece de procedimientos precisos para darles cumplimiento, lo que supone un importante obstáculo para garantizar el acceso de las mujeres al aborto en casos de violación o cualquier otra circunstancia amparada por las leyes. ${ }^{10}$ Los hospitales públicos que ofrecen servicios de aborto son pocos y se concentran mayormente en el Distrito Federal. Además, las mujeres en general desconocen las leyes y el proceso para autorizar los abortos suele ser complicado, debido al burocratismo de los servicios legales y médicos responsables; es común que la autorización se obtenga cuando el embarazo sobrepasó el primer trimestre y en esos casos la mayoría de los médicos se rehúsa a practicar abortos. ${ }^{11-13}$

La prohibición legal del aborto nunca ha impedido su práctica clandestina, cuyas consecuencias adversas afectan sobre todo a las mujeres de los sectores pobres, que carecen de recursos económicos e interrumpen sus embarazos bajo condiciones de inseguridad. Por ello, uno de los propósitos de la despenalización del aborto es "eliminar la injusticia social que genera la ilegalidad y atenuar los costos humanos, económicos y sanitarios concomitantes". ${ }^{14}$

Una primera reforma para liberalizar las leyes sobre el aborto en México tuvo lugar en el Distrito Federal en 2000. Con esta reforma se ampliaron las causales para el aborto legal, incluyendo, además del embarazo resultado de violación, vigente en todo el país, indicaciones que existían en otros estados: riesgo para la salud de la mujer, malformaciones fetales e inseminación artificial no consentida. ${ }^{15}$ Esta reforma proporcionó una sólida base para ofrecer servicios de aborto legal en los hospitales públicos y el debate sin precedentes que generó propició algunos cambios en los códigos penales de otras entidades federativas. ${ }^{9,11,16, *}$ En 2007, se aprobó una reforma que despenalizó el aborto en la Ciudad de México. El decreto de ley establece que el aborto se permite a solicitud de la mujer hasta las 12 semanas del embarazo y lo autoriza después de ese plazo en casos de violación, grave riesgo para la salud o la vida de la mujer, y malformaciones genéticas o congénitas severas. Los servicios pueden ofrecerse en unidades de los sectores público, social y privado, por ginecólogos-obstetras o cirujanos generales. La ley ordena a la Secretaría de Salud (SSA) del Distrito Federal proporcionar en sus hospitales servicios de aborto, seguros y gratuitos, para las mujeres residentes en la entidad, así como garantizar el acceso a la anticoncepción para prevenir los embarazos no deseados. Al gobierno local corresponde instrumentar políticas para promover la salud sexual, los derechos reproductivos, y la maternidad y paternidad responsables. ${ }^{17,18}$

La despenalización del aborto en el Distrito Federal responde a la necesidad de abatir la alta incidencia de daños ocasionados por el aborto inseguro. En 2005, la razón de mortalidad materna en el país fue de 63.3 muertes por 100000 nacidos vivos; para el Distrito Federal la cifra fue menor, con 57.6, pero mayor a la de otras 15 entidades. Entre 1990 y 2005, el aborto representó la tercera causa de mortalidad materna en el Distrito Federal, mientras en el ámbito nacional constituyó la quinta. En 2005, la tasa anual promedio de hospitalización por aborto por cada mil mujeres de 15 a 49 años $^{\ddagger}$ en el Distrito Federal era de 9.1, cifra significativamente alta respecto del indicador nacional, que era de 5.8. ${ }^{19}$

Por otro lado, las reformas del Distrito Federal se inscriben en la tendencia mundial hacia la liberalización de las leyes sobre el aborto que existe desde los años ochenta, la cual ha sido un factor clave en la reducción de la mortalidad materna en muchos países. Datos de la Organización Mundial de la Salud (OMS) indican que la mortalidad materna por aborto es mucho mayor en

\footnotetext{
* En 2000 se aprobó en Morelos una iniciativa similar a la del Distrito Federal. ${ }^{14}$ En 2003 el código penal de Veracruz añadió la inseminación artificial no consentida y las malformaciones fetales. En 2005 en Baja California Sur se agregó el riesgo para la salud de la mujer y se reglamentó el aborto por violación. En 2006 se publicó un nuevo código penal en Chihuahua que incluyó la inseminación artificial no consentida y eliminó el castigo para el aborto culposo. ${ }^{16}$

₹ Se refiere al número de egresos hospitalarios por aborto en mujeres de 15 a 49 años por cada mil mujeres del mismo grupo de edad. Tasa calculada por Ipas México a partir de información oficial. ${ }^{19}$
} 
las regiones y países con leyes restrictivas; en América Latina ocurren 30 muertes por aborto por cada 100000 nacidos vivos, mientras en Europa se registran sólo cinco. ${ }^{20}$ En casi todos los países con leyes liberales la política gubernamental asegura el acceso a servicios de aborto; la mayoría de los abortos se practica en el primer trimestre del embarazo, por personal capacitado y con procedimientos quirúrgicos o médicos seguros. ${ }^{21-23}$

Si bien la legalidad juega un papel decisivo para prevenir las consecuencias del aborto inseguro, la liberalización de las leyes no garantiza por sí sola que el aborto seguro es accesible para todas las mujeres. Las reformas tienen un impacto limitado o nulo cuando las leyes carecen del apoyo político necesario; y cuando los servicios de aborto son escasos y las mujeres no saben que existen o enfrentan dificultades para obtenerlos. El impacto puede ser también limitado cuando los médicos no entienden las leyes, carecen de capacitación para practicar abortos o se rehúsan a ello por temor a ser procesados penalmente, o bien cuando por motivos morales o religiosos se acogen a las cláusulas de objeción de conciencia incluidas en las leyes.22-24

La ley que despenalizó el aborto en el Distrito Federal entró en vigor en abril de 2007 y para diciembre de ese año el Programa de Interrupción Legal del Embarazo que opera en los hospitales de la SSA del Distrito Federal había atendido a poco más de 4000 mujeres. La nueva ley tiene el pleno respaldo político del gobierno local, pero los hospitales que ofrecen servicios de aborto requieren contar con personal médico suficiente y capacitado, tanto para continuar respondiendo a la demanda inmediata como para asegurar la viabilidad de la reforma a largo plazo. El 70\% de los médicos se ha negado a ofrecer servicios de aborto por objeción de conciencia y sólo una minoría ha participado en los cursos de capacitación impartidos en los hospitales. ${ }^{*}$

\section{Formación de los médicos}

Aplicar el enfoque de la SSR supone considerar los determinantes socioeconómicos y culturales de la salud; reconocer los factores que limitan la autonomía de las mujeres y promover la equidad de género; construir un marco institucional de respeto a los derechos sexuales y reproductivos; superar los problemas logísticos que dificultan la operación de los servicios y formar personal con capacidad para transformarlos. ${ }^{2,3}$

\footnotetext{
* Información proporcionada por la Dirección de Atención Hospitalaria, SSA del Distrito Federal (28 de noviembre de 2007).
}

Frente a este reto, sin embargo, se ha documentado que en muchos países en desarrollo el interés por ampliar las perspectivas de la educación médica en el campo de la SSR es reciente, y que las recomendaciones de la CIPD han tenido poca influencia en los programas de formación básica, postgrado y educación continua para médicos y otros profesionales de la salud. ${ }^{25} \mathrm{En}$ el contexto latinoamericano el enfoque de la SSR y el tema de los derechos sexuales y reproductivos han empezado a ganar espacio en el ámbito universitario, pero se desconocen aún los alcances de su incorporación en distintos programas educativos, incluidos los de algunas escuelas de medicina. ${ }^{26}$

Algunos organismos internacionales han señaladola necesidad de mejorar la calidad de la educación médica en los ámbitos de la SSR y la atención del aborto. La Organización Panamericana de la Salud (OPS), por ejemplo, realizó a principios de los años noventa reuniones para analizar la enseñanza de la SSR en las universidades latinoamericanas, cuyas recomendaciones incluyeron integrar las ciencias sociales a la docencia en esta área, reforzar los componentes éticos en la educación médica, incorporar la anticoncepción postaborto al currículum, y una mayor relación entre las universidades y los servicios de salud. ${ }^{27-29}$ La Federación Internacional de Ginecología y Obstetricia (FIGO) realizó en 1997, con apoyo de la OMS, un encuentro regional latinoamericano en el que se abordó la responsabilidad de los ginecólogos-obstetras hacia el aborto y se enfatizó la necesidad de incorporar un debate amplio sobre el tema en las sociedades médicas, los servicios de salud y las universidades. A las sociedades de ginecólogos-obstetras se recomendó contribuir al mejoramiento de la educación médica en los aspectos relacionados con el aborto y el embarazo no deseado. ${ }^{30}$ En el ámbito nacional, la Federación Mexicana de Ginecología y Obstetricia (Femego) realizó en el 2000 -en colaboración con la FIGO, organizaciones no gubernamentales, instituciones de salud, universidades y sociedades médicas-reuniones para analizar las repercusiones sociales y médicas de la violencia sexual, cuyas recomendaciones incluyeron la formación de personal para ofrecer a las víctimas una atención integral, así como su capacitación en modelos para interrumpir el embarazo en casos de violación. ${ }^{31,32}$

A pesar de estas recomendaciones y del esfuerzo conjunto de algunos grupos académicos y organizaciones no gubernamentales con experiencia en la capacitación de personal en los servicios, las iniciativas en México para mejorar la educación médica en los ámbitos de la SSR y la atención al aborto han sido escasas. De acuerdo con la Asociación Mexicana de Facultades y Escuelas de Medicina (AMFEM), la educación médica debe contribuir a mejorar la calidad de la atención 
médica que se ofrece en el país y a elevar el bienestar de la población. ${ }^{33}$ Sin embargo, desde esta Asociación, responsable de vigilar la calidad de la educación médica que ofrecen las universidades, poco se ha hecho para que la formación de los médicos tenga una mayor coherencia con las necesidades sociales y las políticas públicas. Una revisión de los planes de estudio de 11 escuelas públicas de medicina, realizada en $2001,{ }^{1}$ permitió observar que los propósitos de la AMFEM están lejos de ser alcanzados a partir del modelo que predomina en la educación médica. * Además, la revisión de los programas de ginecología y obstetricia de esas escuelas mostró que en todas existía, en mayor o menor medida, la necesidad de introducir cambios para incorporar o lograr una mejor integración de los aspectos relacionados con la SSR y la atención del aborto.

Desde la mirada feminista y en contextos distintos al de México, grupos académicos han elaborado propuestas para mejorar la educación médica en el ámbito de la salud de la mujer, que parten del cuestionamiento a los sesgos masculinos que predominan en las ciencias biomédicas y la práctica de la medicina, así como de la crítica al poder que habitualmente ejercen los médicos sobre las mujeres. Estas propuestas se dirigen a superar la visión centrada en la reproducción biológica y a construir un enfoque multidisciplinario sustentado en los problemas que afectan a las mujeres a lo largo de su ciclo vital; a incorporar nuevas áreas temáticas, como los estudios de género y la perspectiva multicultural; a crear asignaturas optativas, cursos interdisciplinarios y currículos integrados; y a introducir herramientas didácticas que favorezcan un aprendizaje más activo. ${ }^{34-42}$ Como parte de esta corriente se han desarrollado iniciativas en el campo de la SSR, ${ }^{\ddagger}$ que buscan incluir nuevos enfoques

\footnotetext{
* En la mayoría de las universidades el currículum responde a un modelo que privilegia los aspectos biomédicos y curativos, fragmenta el conocimiento en disciplinas aisladas y se orienta a la práctica hospitalaria especializada. El modelo privilegia la enseñanza de las disciplinas básicas y clínicas, otorgando menor importancia a las relacionadas con las ciencias sociales y la salud pública, así como a la formación de los estudiantes en espacios comunitarios y servicios de primer nivel. La docencia se apoya en general en modelos pedagógicos que limitan la integración del conocimiento y el desarrollo de la capacidad de reflexión crítica en los estudiantes.

₹ Un ejemplo es la iniciativa de la Association of Reproductive Health Professionals (ARHP), que en 2005 dio a conocer una propuesta curricular en SSR (Curriculum Initiative for Reproductive Health) que se usa en escuelas de medicina, residencias de ginecología y obstetricia, y programas de formación médica avanzada (www. arhp.org) La ARHP apoya las actividades de una organización estudiantil, Medical Students for Choice, con influencia en Estados Unidos y Canadá, cuyo propósito es lograr que el aborto se incluya en los programas de formación médica (www.ms4c.org). ${ }^{41}$
}

para abordar la sexualidad y la reproducción, y ampliar los espacios curriculares para incorporar temas como el aborto, la consejería en sexualidad y anticoncepción, los derechos sexuales y reproductivos, la violencia sexual, y la sexualidad adolescente, entre otros. ${ }^{25,41,42}$

Las razones por las cuales aspectos importantes de la salud de las mujeres están ausentes en los currículos de formación médica han sido señaladas por distintos autores e incluyen la resistencia para cambiar la orientación y la estructura de los planes de estudio; la sobrecarga de contenidos, no siempre útiles, en los programas de estudio; la competencia con otras prioridades curriculares; la carencia de personal docente capacitado; los sesgos de género; o la percepción de que estos aspectos carecen de interés para la educación médica. ${ }^{25,34-36,40-42}$

La docencia relacionada con la SSR y el aborto es en general impartida por especialistas en ginecología y obstetricia, lo cual presenta algunas dificultades. Se ha señalado, por ejemplo, que esta disciplina ofrece elementos esenciales para la formación clínica de los médicos, pero carece de una perspectiva amplia sobre las necesidades de salud de las mujeres; los ginecólogos-obstetras han sido legitimados como los expertos en el campo de la reproducción, aunque en general su formación les ofrece pocos elementos para ofrecer consejería en aspectos relacionados con la sexualidad. ${ }^{25}$ Se ha planteado también que la ginecología y la obstetricia constituyen un área de la medicina en la que el sexismo y el control sobre las mujeres se expresan con gran claridad, tanto en las relaciones de poder que establecen médicos y pacientes como en la medicalización extrema de procesos como el embarazo, el parto o la menopausia; el abuso de poder es frecuente en esta especialidad y no es raro que los profesionales omitan información importante para la toma de decisiones de sus pacientes, o que realicen intervenciones sin llevar a cabo un proceso adecuado de consentimiento informado. ${ }^{43-45}$

Por otro lado, los ginecólogos-obstetras suelen tener posturas conservadoras frente al aborto, pero dentro de este gremio hay posturas diversas y existen asociaciones profesionales con un enfoque progresista; un ejemplo es la FIGO, cuyo Comité de Ética reconoce explícitamente el derecho de las mujeres al aborto seguro. Sin embargo, en países como los latinoamericanos, que en su mayoría tienen leyes restrictivas y la religión católica ejerce una gran influencia, es común que los ginecólogos-obstetras se opongan al aborto; hay especialistas que lo justifican en algunas circunstancias, aunque raramente lo aceptan frente a sus colegas y pocos están preparados para practicarlo. La mayoría considera que la capacitación en procedimientos para interrumpir el embarazo es poco útil en términos de su prestigio y desarrollo como 
profesionales, aunque es un hecho que muchos se entrenan en ellos con fines de lucro. En este contexto, pocos ginecólogos-obstetras tienen una comprensión cabal de los problemas relacionados con el aborto y sólo una minoría lo practica desde una postura ética apoyada en el respeto a la autonomía de sus pacientes. ${ }^{46}$

Las resistencias para incorporar el aborto en la educación médica no son exclusivas de los países con leyes restrictivas. En Estados Unidos, por ejemplo, el aborto es legal y representa una de las intervenciones quirúrgicas más comunes, pero no está incluido en el currículum de la mayoría de las escuelas de medicina. ${ }^{47}$ Además, pocas residencias en ginecología y obstetricia ofrecen capacitación en procedimientos para interrumpir el embarazo y la mitad de los especialistas se gradúan con poca o ninguna experiencia en su manejo. Esta situación, vinculada al movimiento anti-choice que existe en ese país, ha provocado una disminución significativa de los médicos capacitados y dispuestos a ofrecer servicios de aborto. $^{48,49}$

En México la formación en ginecología y obstetricia ofrece una perspectiva limitada para el tratamiento de las complicaciones del aborto. La aspiración manual endouterina (AMEU), que es el procedimiento recomendado por la OMS para tratar este tipo de complicaciones, ${ }^{50}$ tanto en casos de abortos espontáneos como electivos, está ausente en prácticamente todas las escuelas de medicina. ${ }^{*}$ Tal carencia es difícil de justificar, ya que la AMEU es un procedimiento cuya eficacia y seguridad han sido avaladas por numerosos estudios y que ha desplazado en muchos lugares al legrado uterino instrumental. En 2000, la SSA publicó un documento normativo en el que la AMEU es recomendada como la primera opción para tratar la hemorragia obstétrica y el aborto incompleto ${ }^{51}$ Además, la AMEU forma parte del modelo de atención postaborto (APA), que empezó a usarse en los años 90 en países donde las complicaciones del aborto se asocian con niveles elevados de mortalidad materna..$^{52}$ Las instituciones públicas han acumulado experiencias de capacitación de personal en el modelo de la APA con AMEU en muchos hospitales del país, y se han operado también programas para introducirlo en unidades del primer nivel en el medio rural. ${ }^{\ddagger}$ Entre los beneficios del modelo se han reportado un mayor nivel

\footnotetext{
* La única excepción es la facultad de Medicina de la Universidad Nacional Autónoma de México (UNAM), que incluyó la AMEU en los contenidos de su programa de Internado Médico. ${ }^{1}$

* Secretaría de Salud, Instituto Mexicano del Seguro Social, Programa IMSS-Oportunidades e ISSSTE. En el primer nivel de atención el modelo se ha usado en el Programa IMSS-Oportunidades y en unidades de la SSA en el estado de Michoacán. ${ }^{55}$
}

de satisfacción entre usuarias y proveedores, el mejoramiento de la calidad técnica y humana de los servicios, el aumento del número de mujeres que reciben consejería, y la disminución de las estancias hospitalarias y los costos de atención. ${ }^{52-55}$ En los últimos años, sin embargo, los recursos para expandir este modelo han disminuido y la atención a las mujeres con complicaciones del aborto continúa siendo, en general, de baja calidad; el legrado intrauterino instrumental se usa todavía en muchos servicios y un número importante de mujeres egresan sin recibir consejería amplia en anticoncepción. ${ }^{56}$

Los procedimientos de elección para la interrupción del embarazo, que en la actualidad incluyen la AMEU y el aborto con medicamentos -inducido con fármacos como la mifepristona y el misoprostol-, tampoco están incluidos en los programas de estudio. Una publicación reciente de la OMS indica que el legrado uterino instrumental, usado aún en muchos lugares para interrumpir embarazos, debe utilizarse solamente cuando la AMEU o el aborto con medicamentos no estén disponibles. ${ }^{50} \mathrm{El}$ aborto con medicamentos ha empezado a ganar atención entre los médicos de distintos países de América Latina. El medicamento más accesible en la región es el misoprostol, indicado para tratar la úlcera péptica pero cuyo uso es común en la obstetricia para inducir el parto, tratar abortos retenidos e interrumpir embarazos. Existen evidencias de que el misoprostol es ampliamente utilizado sin prescripción médica para interrumpir embarazos y su uso con este fin se ha asociado con un menor índice de complicaciones. . $^{57,58}$

Los programas de ginecología y obstetricia incluyen contenidos de planificación familiar, pero en general omiten o dan poca importancia a aspectos centrales para mejorar la calidad de los servicios e incidir más eficazmente en la prevención de los embarazos no deseados, como la consejería y el consentimiento informado, o la anticoncepción para adolescentes. Otro importante vacío en estos programas es la anticoncepción hormonal de emergencia, recomendada por la OMS como un método seguro y eficaz para prevenir los embarazos no deseados y los abortos, ${ }^{59} \mathrm{e}$ incluida en la Norma Oficial Mexicana de los Servicios de Planificación Familiar desde 2004. ${ }^{60}$

La información sobre los aspectos legales del aborto que se transmite en las escuelas de medicina no favorece la comprensión adecuada de las leyes y contribuye a fomentar los estigmas morales sobre la interrupción del embarazo. No es raro que en los textos de medicina legal se aluda al aborto inducido como "aborto criminal" y que se califique a quienes lo practican con términos como el de "abortadores profesionales". ${ }^{61}$

La bioética ha empezado a formar parte de las asignaturas de ética médica en algunas escuelas de me- 
dicina, pero en la mayoría los temas de esta disciplina se abordan desde un enfoque que no favorece la reflexión racional sobre problemas que suponen importantes dilemas para los médicos, como el aborto, la eutanasia, la reproducción asistida o la clonación, entre otros. Frente a esta perspectiva, la formación de los médicos tiene que incorporar el amplio bagaje de publicaciones producidas por autores mexicanos que comparten una postura liberal y laica para analizar los problemas del campo de la bioética. ${ }^{*}$ En el caso del aborto se parte, en general, de un enfoque apoyado en valores y dogmas religiosos. Un ejemplo de este enfoque se encuentra en un libro de bioética para estudiantes de medicina -avalado por la AMFEM y la Academia Nacional de Medicina-, en el que el aborto es equiparado, sin matices y bajo cualquier circunstancia, con el homicidio. ${ }^{62} \mathrm{El}$ aborto es un tema central del debate bioético actual y en México existen publicaciones que contribuyen tanto a la reflexión sobre los dilemas morales que genera como a la discusión crítica e informada de las leyes que lo regulan. Un debate racional sobre el tema en las escuelas de medicina requiere un enfoque que abarque las distintas posturas éticas sobre el aborto; que permita comprender la diversidad de códigos morales que coexisten en la sociedad mexicana; y que reconozca la capacidad de las mujeres para ejercer sus derechos y decidir en torno a la sexualidad y la reproducción. .3,64 $^{2}$

La atención que reciben las mujeres con complicaciones del aborto es un buen ejemplo de la influencia que ejercen los estigmas morales en las posturas de los médicos frente a la interrupción del embarazo. Para muchas mujeres, la experiencia suele ser negativa y en ocasiones francamente traumática, ya que frente a la sospecha, no siempre fundada, de que el aborto fue intencional, es común que sean objeto de actitudes hostiles y punitivas por parte de quienes las atienden. ${ }^{46,65-67} \mathrm{Se}$ ha planteado que en el maltrato hacia las mujeres que abortan influyen elementos que rebasan la esfera individual, en tanto los comportamientos deshumanizados, frecuentes en la práctica de la medicina, son propiciados

\footnotetext{
* Una institución mexicana dedicada a la construcción del enfoque bioético liberal y laico es el Colegio de Bioética, creado en 2003 como respuesta al dogmatismo de la Academia Mexicana de Bioética y a las posiciones de condena moral adoptadas por sectores como la iglesia católica y Pro-Vida frente a asuntos del campo de la bioética (www.colbio.org.mx). La Facultad de Medicina de la UNAM ha empezado a incorporar esta perspectiva a sus programas de ética, pero la mayor parte de las actividades académicas para difundir el enfoque bioético liberal y laico se llevan a cabo en otros espacios de la UNAM, como el Instituto de Investigaciones Jurídicas y el Instituto de Investigaciones Filosóficas.
}

y legitimados a partir de una determinada estructura política, institucional o profesional; los individuos asumen y reproducen sistemas particulares de valores que se transmiten en la relación docente-estudiante y que más tarde se expresan en la relación médico-paciente. ${ }^{68}$ Por otro lado, el maltrato en estos casos tiene que ver no sólo con la prohibición legal del aborto o con una percepción moldeada por patrones culturales y reforzada por la educación médica, sino también con aspectos ligados a la subordinación genérica de las mujeres, a la pobreza de quienes sufren complicaciones con mayor frecuencia, y a las relaciones de poder que habitualmente se establecen entre médicos y pacientes:

el aborto pone en funcionamiento una serie de reacciones profundamente enraizadas en la subjetividad de los agentes de salud, fundadas en modelos ideológicos referidos a la maternidad y la sexualidad, a la vez que reactualiza prejuicios de clase y refuerza relaciones de poder propias de la atención médica en nuestra sociedad. En este sentido, la intervención técnica está ligada a una función de control social, de modo que la función punitiva no ha sido ni es ajena a la práctica médica. ${ }^{66}$

Muchos médicos eminentes en México han apoyado el movimiento para liberalizar las leyes sobre el aborto, pero la gran mayoría se ha mantenido al margen del debate público sobre el tema. Los resultados de las encuestas aplicadas a médicos en el país sustentan la necesidad de ampliar las perspectivas de la educación médica en relación con el aborto; la mayoría de los médicos acepta su legalidad en casos de violación y por razones médicas, pero el consenso es menor frente a otro tipo de circunstancias. ${ }^{8,11,69} \mathrm{La}$ encuesta más reciente se aplicó en 2002 a una muestra de 1206 profesionales -ginecólogos-obstetras, médicos familiares y generales- que trabajaban en clínicas y hospitales de la SSA y el sector privado en áreas urbanas del país. El 93\% opinó que el aborto debe ser legal cuando la vida de la mujer está en riesgo; para casos de violación, riesgo para la salud de la mujer y alteraciones fetales las proporciones oscilaron alrededor del 85 por ciento. La aceptación fue menor en otras circunstancias, con $56 \%$ para casos de inseminación artificial no consentida; sólo 20\% aceptó el aborto por decisión de la mujer y los porcentajes fueron aun menores en casos de falla anticonceptiva, falta de recursos económicos, mujeres menores de edad y madres solteras. La mayoría, con $84 \%$, opinó que los hospitales públicos deberían ofrecer servicios de aborto en los casos previstos en las leyes, pero sólo poco más de la mitad indicó que estaría dispuesta a ofrecer intervenciones legales. ${ }^{69}$ 


\section{Conclusiones}

La formación de personal capacitado para atender las necesidades de SSR de la población mexicana debe recibir mayor atención en las escuelas de medicina, ya que el éxito de los programas institucionales en esta área no depende sólo de contar con mayores recursos financieros o con una mejor infraestructura de servicios. Puede afirmarse, de hecho, que el impacto limitado de muchos programas de SSR y los problemas de calidad de los servicios se deben en buena medida a deficiencias de la educación médica.

En los acuerdos de la CIPD el aborto seguro fue definido como un componente esencial de los servicios de SSR, por lo que los compromisos internacionales firmados por México, así como las recomendaciones de organismos como la OPS, la FIGO y la Femego, ofrecen una sólida base para capacitar a los futuros médicos en el modelo de la APA y en las opciones que ofrecen mayor seguridad para la interrupción del embarazo.

Las instituciones públicas de salud han destinado una gran cantidad de recursos para sensibilizar y capacitar al personal médico de los servicios, los cuales podrían ser optimizados si las escuelas de medicina ofrecieran una mejor formación en sus licenciaturas. Al igual que las instituciones de salud, las universidades podían establecer relaciones de colaboración con las organizaciones no gubernamentales que trabajan en los ámbitos de la SSR y de la atención al aborto, y que han acumulado una gran experiencia en la capacitación de personal.* $^{*}$

La despenalización del aborto en el Distrito Federal representa un importante desafío para los servicios de salud y las posibilidades de lograr cabalmente sus propósitos dependerán en buena medida de contar con médicos dispuestos a ofrecer a las mujeres servicios legales y seguros. Por su condición privilegiada en la jerarquía de los servicios, los médicos ejercen una gran influencia en las actitudes que frente al aborto y otros problemas del campo de la SSR tienen otros profesionales de la salud. Por ello, corresponde a los médicos promover un debate amplio sobre sus responsabilidades frente a la interrupción del embarazo al interior de las escuelas de medicina, los servicios de salud y las asociaciones médicas. Corresponde también a los médicos asegurar su derecho a trabajar con los recursos necesarios para

\footnotetext{
* Dos organizaciones que han colaborado con las instituciones de salud para capacitar personal en los modelos de atención integral del aborto son Ipas México (www.ipas.org) y Population Council (www.popcouncil.org).
}

garantizar el acceso de las mujeres al aborto legal y ofrecer una atención respetuosa y con calidad a quienes sufren complicaciones del aborto inseguro.

Incorporar un enfoque integral sobre las distintas dimensiones del aborto en las escuelas de medicina implica elaborar propuestas apoyadas en evidencias sólidas. En tal sentido, es necesario desarrollar proyectos de investigación que permitan identificar los rezagos y avances en la incorporación de contenidos sobre SSR y aborto en el currículum de las facultades de medicina, así como evaluar el impacto de las intervenciones educativas dirigidas a estudiantes. Un asunto que merece ser atendido con urgencia es la formación de profesores capacitados para la docencia en SSR, a partir de modelos curriculares que les proporcionen una perspectiva actualizada de los problemas y necesidades en este campo.

Las propuestas para ofrecer a los estudiantes de medicina una perspectiva amplia sobre el aborto provocan resistencias y son en general percibidas como una amenaza a las concepciones que dominan en la profesión médica. Modificar las actitudes de los médicos frente a problemas como el aborto supone llevar a cabo una tarea difícil, cuyos resultados no se percibirán en el corto plazo. De cualquier manera, es importante insistir en la necesidad de promover cambios curriculares orientados a garantizar que la educación médica contribuya al mejoramiento de las condiciones de salud de las mujeres y de la población mexicana en general.

\section{Agradecimientos}

A la Dra. Nadine Gasman, ex directora de Ipas México, por su entusiasmo para la creación del proyecto de colaboración con las universidades y por su decidido apoyo a nuestro trabajo. Agradecemos también el apoyo financiero de la Fundación David and Lucile Packard.

\section{Referencias}

I. González de León D, Billings D, Gasman N, Ramírez R. Incorporación de la salud sexual y reproductiva y del enfoque de la atención integral a la mujer con aborto en los programas de licenciatura en medicina. México: Ipas México, 2002.

2. Langer A. Salud sexual y reproductiva: dónde estamos a casi una década después de El Cairo. En: Bronfman M, Delamn C, comp. Salud reproductiva. Temas y debates. México: Instituto Nacional de Salud Pública, 2003:25-34.

3. Langer A. Planificación familiar y salud reproductiva o planificación vs salud reproductiva. Desafíos para llevar el paradigma de la salud reproductiva de la retórica a la práctica. En: Bronfman M, Castro R, comp. Salud, cambio social y políticas. Perspectivas desde América Latina. México: Instituto Nacional de Salud Pública / EDAMEX, 1999:135- 149. 
4. Asamblea General de las Naciones Unidas. Programa de Acción de la Conferencia Internacional sobre Población y Desarrollo. Documento A/I7I//3. Nueva York: ONU, 1994.

5. Asamblea General de las Naciones Unidas. Medidas clave para seguir ejecutando el Programa de Acción de la Conferencia Internacional sobre Población y Desarrollo. Nueva York: ONU, 1999.

6. Secretaría de Salud. El derecho a la libre decisión. La planificación familiar en el contexto de la salud reproductiva. México: SSA, Subsecretaría de Prevención y Control de Enfermedades / Dirección General de Salud Reproductiva, 1998.

7. Secretaría de Salud. Programa de Acción: Salud Reproductiva. México: SSA, 2001.

8. González de León D, Billings DL.Attitudes towards abortion among medical trainees in México City public hospitals. Gend Dev 2001;9(2): 87-94.

9. Lamas M, Bissell S. Abortion and politics in Mexico: context is all. Reprod Health Matters, 2000;8(I6): 10-23.

10. Grupo de Información en Reproducción Elegida. Leyes sobre el aborto en México. Hoja informativa. México: GIRE, 2007.

II. Billings DL, Moreno C, Ramos C, Gonzalez de Leon D, Ramirez R, Villaseñor $L$, et al. Constructing access to legal abortion services in Mexico City. Reprod Health Matters 2002;10(19):86-94.

12. Becker D, Garcia S, Larsen U. Knowledge and opinions about abortion law among Mexican youth. Int Fam Plan Perspect 2002;28(4):205-213. 13. Lara D, García S, Strickler J, Martínez H,Villanueva L. El acceso al aborto legal de las mujeres embarazadas por violación en la Ciudad de México. Gac Med Mex 2003;139(Supl I):77-90.

14. Lamas M.Aborto: entre el no de la religión y el sí de las mujeres. En: Bronfman M, Denman C, comp. Salud reproductiva. Temas y debates. México: Instituto Nacional de Salud Pública, 2003.

15. Asamblea Legislativa del Distrito Federal. Decreto por el que se adicionan diversas disposiciones del Código Penal para el Distrito Federal y el Código de Procedimientos Penales para el Distrito Federal. Gaceta Oficial del Distrito Federal, I0ª época 2000; I48, 24 de agosto.

16. Grupo de Información en Reproducción Elegida. Cronología de la despenalización del aborto en México. México: GIRE. [Consultado 7 de julio 2007]. Disponible en: www.gire.org.mx.

17.Asamblea Legislativa del Distrito Federal. Decreto por el que se reforma el Código Penal para el Distrito Federal y se adiciona la Ley de Salud para el Distrito Federal. Gaceta Oficial del Distrito Federal, $17^{\mathrm{a}}$ época 2007;70, 26 de abril.

18.Administración Pública del Distrito Federal / Secretaría de Salud. Acuerdo que reforma, adiciona y deroga diversos puntos de la circular GDF-SSDF/0I/06 que contiene los lineamientos generales de organización y operación de los servicios de salud relacionados con la interrupción del embarazo en el Distrito Federal. Gaceta Oficial del Distrito Federal, $17^{\mathrm{a}}$ época 2007;75, 4 de mayo.

19. Schiavone R, Troncoso E, Bilings D, Polo G. Abortion related morbidity in public sector hospitals in Mexico, 2000-2005. Seminar on Measurement of Abortion Incidence, Abortion-related Morbidity and Mortality. Paris, France: International Union for the Scientific Study in Population, 2007. 20.World Health Organization. Unsafe abortion: global and regional estimates of the incidence and mortality due to unsafe abortion with a listing of available country data. Geneva:WHO, 2004.

21. Berer M. Making abortion a woman's right worldwide. Reprod Health Matters 2002;10(19): I-8.

22. Warriner IK. Unsafe abortion: an overview of priorities and needs. In:Warriner IK, ed. Preventing unsafe abortion and its consequences. Priorities for research and action. New York: Guttmacher Institute, 2006. 23. Cook R, Dickens BM, Fathalla MF. Reproductive health and human rights: integrating medicine, ethics, and law. New York: Oxford University Press, 2003.

24. Rahman A, Katzive L, Henshaw SK.A global review of laws on induced abortion. Fam Plann Perspect 1998;24(2);56-64.
25. Haslegrave M, Olatunbosun O. Incorporating sexual and reproductive health in the medical curriculum in developing countries. Reprod Health Matters 2003; II (2I):49-58.

26. Cardaci D. Enfoque de género y derechos sexuales y reproductivos en el currículum de medicina. Análisis de experiencias identificadas en América Latina y El Caribe. México: Fondo de Población de las Naciones Unidas, 2002.

27. Organización Panamericana de la Salud. Taller internacional sobre la planificación familiar post-parto y post-aborto. Relato final. Quito: OPS, 1993.

28. Organización Panamericana de la Salud. Reunión para consolidar la metodología de análisis prospectiva en salud reproductiva y población para escuelas de ciencias de la salud. Caracas: OPS, 1993.

29. Organización Panamericana de la Salud. Marco de referencia para la enseñanza de la salud reproductiva. Washington: OPS / FPNU, 1994. 30. Grupo de Trabajo Federación Internacional de Ginecología y Obstetricia / Organización Mundial de la Salud. Aborto: una responsabilidad profesional de los obstetras y ginecólogos. Campinas, SP, Brasil: Centro de Pesquisas Materno-Infantis de Campinas, 1997.

31. Granados S, Mariscal JD, Campos L. Declaración de Monterrey 2000 sobre violencia familiar y sexual hacia la mujer. Monterrey, Nuevo León: Ipas México, 200I.

32. Uribe R, Billings DL, eds.Violencia sobre la salud de las mujeres, ipor qué hoy? Memoria de la Reunión Internacional de la Federación Mexicana de Ginecología y Obstetricia. México: FEMEGO / Ipas México, 2003.

33. Asociación Mexicana de Facultades y Escuelas de Medicina. Sistema de educación médica. México:AMFEM, sf. [Consultado 4 julio 2007]. Disponible en:www.amfem.edu.mx.

34. Nelson M, JoDean N, Jonson K. Integration or evolution: women's health as a model for interdisciplinary change in medical education. Acad Med 1997;72(9):737-740.

35. Donghue G.Women's health: a catalyst for reform of medical education.Academic Medicine 2000;75(II): 1056-1060.

36. Núñez AE. Transforming cultural competence into cross-cultural efficacy in women's health.Acad Med 2000;75(II):107I-1080.

37. Krasnoff M. Resources for teaching about women's health.Acad Med 2000;75(I I): 1087-1094.

38. Phillips SP. Evaluating women's health and gender. Am J Obstet Gynecol 2002; 187:S22-S24.

39. Stalburg CM, Stein TA.An interdisciplinary course in women's health integrating basic and clinical sciences: clinical anatomy and women's health. Am J Obstet Gynecol 2002;187: 49-52.

40. Autry AM, Meurer LM, Barnabei VM, Green SS, Johnson-Masotti AP, Otto-Salaj LL, et al.A longitudinal women's health curriculum: a multi method, multiperspective needs assessment. Am J Obstet Gynecol 2002; 187:SI2-SI4.

4I. Lazarus CJ, Brown S, Doyle LL. Securing the future: a case for improving clinical education in reproductive health. Contraception 2007;75:8I-83.

42. Thomas M, Shields WC. Leadership and diversity: a call for new directions in reproductive health education and practice. Contraception 2007;75: 163-165.

43. Castro R, Bronfman M. Teoría feminista y sociología médica: bases para una discusión. En: Figueroa JG, comp. La condición de la mujer en el espacio de la salud. México: El Colegio de México 1998: 205-230. 44. Rosas, CF, coord. Ética em ginecología e obstetricia. $3^{a}$ Ed. Sao Paulo: Conselho Regional de Medicina do Estado de Sao Paulo, Colec. Cuadernos CREMESP, 2004.

45. Scully D. Men who control women's health. The miseducation of obstetrician-gynecologists. New York:Teachers College Press, 1994. 46. Faúndes A. Barzelatto J. El drama del aborto. En busca de un consenso. Bogotá:Tercer Mundo Editores, 2005.

47. Espey E, Ogburn T, Chavez A, Qualis C, Leyva M.Abortion education in medical schools: a national survey.Am J Obstet Gynecol 2005;192:640-643. 
48. Joffe C. Doctors of conscience. The struggle to provide abortion before and after Rode v.Wade. Boston: Beacon Press, 2001. 49. Donohoe M. Increase in obstacles to abortion: the American perspective in 2004. J Am Med Womens Assoc 2005;60:16-25. 50. Organización Mundial de la Salud.Aborto sin riesgos. Guía técnica de políticas para sistemas de salud. Ginebra: OMS, 2003.

51. Secretaría de Salud. Lineamiento técnico para la prevención y manejo de la hemorragia obstétrica. México: SSA, Dirección General de Salud Reproductiva, 2000.

52. Billings DL, Crane BB, Benson J, Solo J, Fetters T. Scaling up a public health innovation: a comparative study of post-abortion care in Bolivia and Mexico. Soc Sci Med 2007;64(I I):221 10-2222.

53. Brambila C, Langer A, García C, Heimburger A. Estimating costs of post-abortion services at Doctor Aurelio Valdivieso General Hospital, Oaxaca, Mexico. In: Huntington D, Piet-Pelon NJ, eds. Post-abortion care: lessons from operation research. New York: Population Council, 1999 54. Quiroz G, Billings D, Gasman N.Aspiración manual endouterina: tecnología adecuada para la atención de calidad a mujeres en situación de aborto. Gac Med Méx 2003;139(Supl):S65-S73.

55. Billings D, González de León D, Ramírez R. Atención post-aborto: lecciones aprendidas durante una década de experiencia en México. En: Billings D,Vernon R, comp. Avances en la atención post-aborto en América Latina y El Caribe: Investigando, aplicando y expandiendo. México: Population Council / Ipas / El Manual Moderno, 2007.

56. Langer A. El embarazo no deseado y el aborto inseguro: su impacto sobre la salud en México. Gac Med Mex, 2003; 139,SI:3-7.

57. Faúndes A. Uso de misoprostol en obstetricia y ginecología. $2^{\mathrm{a}}$ edición. Santo Domingo, República Dominicana: Federación Latino Americana de Sociedades de Ginecología y Obstetricia, 2007.

58. Lafaurie MM, Grossman D, Troncoso E, Billings D, Chávez S. Women's perspectives on medical abortion in Mexico, Colombia, Ecuador and Peru: a qualitative study. Reprod Health Matters 2005; 3(26):75-83.
59. Organización Mundial de la Salud. Planificación familiar. Un manual mundial para proveedores. Ginebra: OMS/Jons Hopkins Bloomberg School, of Public Health, Center for Communication Programs, 2007. 60. Secretaría de Salud, Norma Oficial Mexicana de los Servicios de Planificación Familiar. México: SSA. [Consultado 7 de julio de 2007]. Disponible en: www.portal.salud.gob.mx.

61. Martínez S, Saldivar L. Medicina Legal. México: Méndez Editores, 2000. 62. Kuthy J,Villalobos JJ, Tarasco M, Yamamoto M, eds. Introducción a la bioética. México: Universidad Anáhuac / Academia Nacional de Medicina / Asociación Mexicana de Facultades y Escuelas de Medicina / Méndez Editores, 2000.

63.Valdés, MM, comp. Controversias sobre el aborto. México: Instituto de Investigaciones Filosóficas, Universidad Nacional Autónoma de México / Fondo de Cultura Económica, 200I.

64. Lamas M. Política y reproducción. Aborto: la frontera del derecho a decidir. México: Plaza y Janés, 200I.

65. Ramos S, Gogna M, Petracci M, Romero M, Szulik D. Los médicos frente a la anticoncepción y el aborto, ¿una transición ideológica? Buenos Aires: Centro de Estudios de Estado y Sociedad, 2001.

66. González Al. Crimen y castigo: el aborto en la Argentina. En: González M (comp). Las mujeres y la salud. México: Programa Interdisciplinario de Estudios de la Mujer, El Colegio de México, 1995: 167- 197.

67. Erviti J. El aborto entre mujeres pobres. Sociología de la experiencia. Cuernavaca, México: Centro Regional de Investigaciones Multidisciplinarias, Universidad Nacional Autónoma de México, 2005. 68. Rance $S$. Trato humano y educación médica: investigación-acción con estudiantes y docentes de la Carrera de Medicina, UMSA. La Paz, Bolivia: Viceministerio de Asuntos de Género, Generacionales y Familia, 1999. 69. Lara D, Goldman L, Firestone M, Garcia S. Opiniones y respuestas. Resultados de una encuesta de opinión a médicos mexicanos sobre el aborto. México: Population Council, 2004. 